\title{
Research on Gait Recognition Method Based on Gait Frame Difference Entropy Image
}

\author{
Zhanli $\mathrm{Li}^{1, \mathrm{a}}$, Fang Yang ${ }^{2, \mathrm{~b}}$, Jingding $\mathrm{Fu}^{3, \mathrm{c}}$, \\ ${ }^{1,2,3}$ Xi'an University of science and technology, 58 Yanta Road, Xi'an, Shannxi, China \\ alizl_xust@163.com, ${ }^{\text {byang10fang@163.com, }{ }^{\mathrm{c}} 455326728 @ q q . c o m}$
}

Keywords: GFDEI, GFDEnI, Shannon entropy, KNN

\begin{abstract}
To the relatively low recognition rate of gait frame difference energy image (GFDEI), this paper proposes gait frame difference entropy image (GFDEnI) to describe gait feature based on it, and then adopts improved KNN to recognize. GFDEnI describes the uncertainty of each point using Shannon entropy theory, and makes the description more accurate. Experimental results show that the algorithm this paper proposes obtains better recognition effect under the three states of ordinary condition, coat wearing and bag wearing, and has certain robustness to human clothing change.
\end{abstract}

\section{Introduction}

Biometric has been payed more and more attention, and the human measurable biometric can make authentication more smart [1]. Recently, gait is viewed as a biological characteristic to feature human and used to solve the human authentication problem. Gait has the advantages of noncontract, distance, difficult to hide, etc, and been a most unique and promising biometrics, and is widely used in security monitoring, virtual reality, etc [2].

Existing gait recognition method can be divided into model based and model free method. Model based approach [3, 4] gets a series of body parameters by establishing human model of arms, legs, and knees, and then obtains the static and dynamic feature from the parameters to recognize [5]. Model free approach does not consider the body's internal structure and establish the priori model. It just statistically analyses the spatiotemporal pattern generated from image sequence, implicitly defines human feature and then extracts gait feature to recognize. Literature [6] proposed a gait recognition method of KPCA and SVM based on gait energy image (GEI), and got a good result by reducing the dimension of feature. GEI can remove the noise of gait image, but loses the relevance information between adjacent frames. Literature [7] viewed gait frame difference energy image (GFDEI) row vector as the gait feature to recognize, which effectively reduces the dimension of features and the amount of computation, but has lower recognition accuracy. Literature [8] adopted gait entropy image (GEnI) as gait feature, which has certain robustness to the change of object's clothing. Literature [9] studied gait recognition based on collaborative representation, solved the small samples and time-consuming problem due to sparse representation, and conducted experiments at multiple perspectives, while the recognition rate is lower at cross perspective.

This paper proposes GFDEnI in a complete gait period based on the Shannon entropy according to the definition of gait entropy image in literature [8]. In an image, the larger the entropy is, the larger the uncertainty of that point is, and the feature change is more obvious. After extracting the gait feature, this paper adopts the nearest neighbor classification to recognize, which was used in literature [10], while literature [10] ignored the different contribution to similarity of different samples. This paper distributes different weight according to the distance between test samples and train samples, and improves the recognition accuracy.

\section{General process of gait recognition}

A complete gait recognition process includes two parts, train and test. Before completing recognition, both train and test process should conduct the operation of gait image preprocess, period test, feature extraction. 
After obtaining the object's gait feature, a gait feature database can be built by train sample and each of them is marked. Then for each test sample, we compare it with the feature saved in the database and get the label to complete recognition.

\section{Preprocessing of gait image}

Images captured in the actual scene will be affected by various factors, which will lead to some defects to image, such as noise, lack of contrast, etc. So we should adopt some relevant preprocess to them.

The image preprocesses this paper uses are moving object detection, morphological processing, binary image normalization and period test. Due to the relatively simple video scene and the almost invariant background, this paper obtains moving object by using background subtraction firstly, and then adopts the operations of binary thresholding, morphology process, such as corrosion and expansion. Thirdly, this paper normalizes the image scale and finally calculates the width of normalized gait silhouette image and draws the period curve. The number of frames between three consecutive maximum or minimum is a complete gait period.

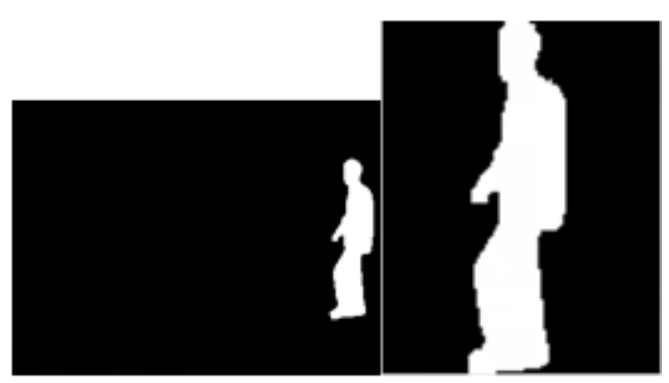

Fig1: Original and normalized image

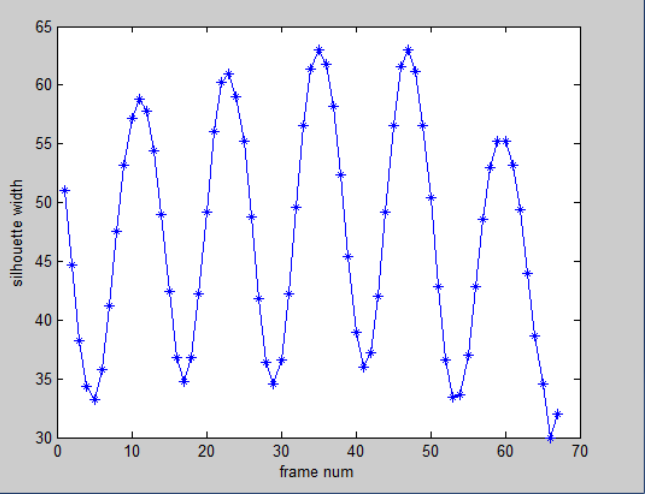

Fig 2: Width change of gait image silhouette with the frame number

\section{Extraction of gait feature}

Generally, people's walk can be viewed as a periodic movement, which includes many human features, such as walking frequency, phase, arm's swing, ankle change. Therefore the gait feature within a period can better describe the human gait characteristic. The common gait features are GEI and GFDEI, etc.

GEI is an average to gait images of a gait period, which reflects the major shape of gait silhouette and the change of these features in a period. GEI not only retains the gait information of silhouette, frequency and phase, reduces the amount of data, but also is not sensitive to noise of single-frame image. For a binary gait image sequence within a given period, GEI is defined as follows:

$$
G(x, y)=\frac{1}{N} \sum_{i=1}^{N} B_{i}(x, y)
$$

Where, $N$ represents the number of image frames in a complete gait period. $t$ represents the current moment in a gait period. $B_{i}(x, y)$ represents the binary silhouette image at $t$ moment in a gait sequence. Figure 3 shows the GEI of the same object under different states.


Fig 3: GEI of the same object in different states

GFDEI describes the related dynamic change information between the adjacent gait images by 
making the difference to two adjacent gait images. GFDEI can suppress the influence of the incomplete silhouette, and maintain most of the shape details. Let $F(x, y, t)$ represents the GFDEI of $t$-th frame, then

$$
F(x, y, t)=F_{a}+F_{b}(t=1,2, \ldots, N)
$$

Where, $F_{a}$ represents the forward GFDI, $F_{b}$ represents the backward GFDI. $N$ represents the depth of the gait image sequence. In this paper, $N$ is taken as the number of gait image within a gait period. Figure 4 shows the GFDEI sequence of the same object under different states.
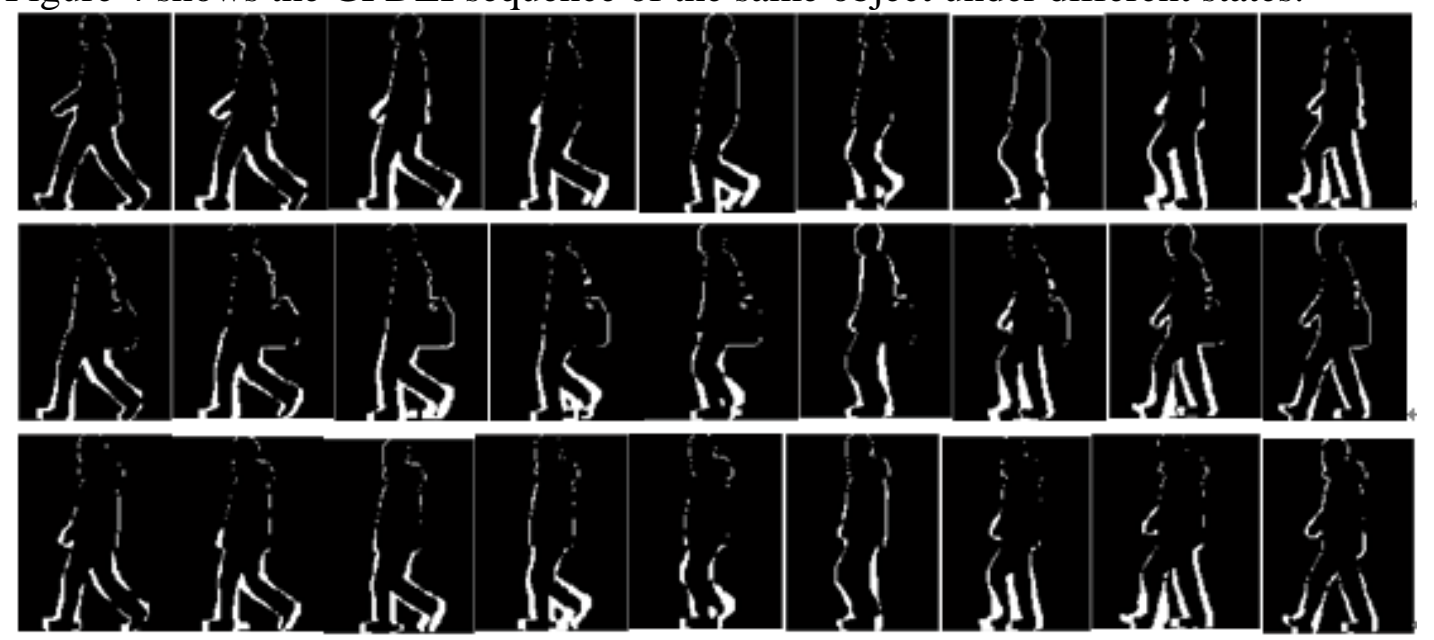

Fig 4: GFDI sequence of the same object under different states (part)

Gait characteristics of the human body are mainly reflected in the changing part of gait image sequences, and the accurate description of changing part can effectively improve the recognition accuracy of gait feature. Shannon entropy is often used to measure the uncertainty of a random variable, and the greater the uncertainty of variables is, the greater the entropy is. In this paper, the entropy is introduced to describe the gait feature, and GFDEnI is proposed based on the GFDEI.

Shannon entropy can be defined by probability theory, as shown (3):

$$
H=-\sum_{i=1}^{n} P_{i} \log \left(P_{i}\right)
$$

Where, $P_{i}$ represents the probability that an event $i$ happens in the $n$ state space.

GFDEnI can be built as follows. In a complete gait period, firstly we obtain the GFDEI sequence by adjacent frame difference, and then get the Shannon entropy of each pixel in the image based on the Shannon entropy principle, and build the GFDEnI.

Let an image of $R \times C$ is defined as $I=[f(x, y)]_{R \times C}$, and $f(x, y)$ is the gray value at $(x, y)$. Let $M_{i}$ represents the number of pixels of gray $i$, then

$$
\sum_{i=0}^{L-1} M_{i}=R C=M
$$

The entropy of an image is defined based on the Shannon entropy, and then

$$
H=-\sum_{i=0}^{L-1} P_{i} \log \left(P_{i}\right) ; \quad P_{i}=\frac{M_{i}}{M}
$$

According to the above theory, we can build the entropy of each pixel in the GFDEnI. 


$$
H(x, y)=-\sum_{i=1}^{N} P_{i}(x, y) \log _{2} P_{i}(x, y)
$$

Where, $P_{i}(x, y)$ represents the probability that $(x, y)$ is at the $n$-th gray.

In this paper, because the gait silhouette image is binary image, we take $\mathrm{N}$ as 2 . After calculating the entropy of each pixel, we adopt Eq. 7 to normalize $H(x, y)$ to ensure it in the range of 0 to 255 . Here, we have finished the process of building the GFDEnI.

$$
G(x, y)=\frac{[H(x, y)-H(\text { min imum })] \times 255}{H(\max \text { imum })-H(\text { min imum })}
$$

Where $H(\min$ imum $)=\min (H(x, y)), H(\max$ imum $)=\max (H(x, y))$.

Figure 5 shows the GFDEnI of the same object under different states.

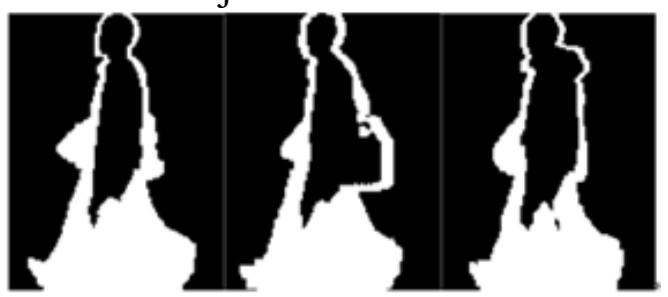

Fig 5: GFDEnI of the same object under different states

\section{Experiment and analysis}

\section{recognition method}

Usually, the method to measure the similarity of the two samples is calculating the Euclidean distance between the two. The calculation formula is as follows:

$$
D_{i j}=\sqrt{\sum_{u=1}^{N}\left(x_{i u}-y_{j u}\right)^{2}}
$$

Where, $x_{i u}$ represents the $u$-th component of the $i$-th sample. $y_{i u}$ represents the $u$-th component of the $j$-th sample. $S$ represents the dimension of the feature space. $D_{i j}$ is the similarity between the two sample features, and the smaller $D_{i j}$ is, the higher the degree of similarity of the two samples is.

In the typical KNN classifier, it is viewed that the $K$ samples have the equal contribution to similarity value, while in fact the impact of different sample to similarity is different and the weight is also different. This paper takes into consideration the weight of Euclidean distance between the test sample and the train sample when calculating the similarity. The closer the distance is, the greater the weight is. On the contrary, the weight is small.

A monotonically decreasing function is needed when determining the weight, this paper views the product of inverse function and exponential function as the weight function, and the expression is as follows:

$$
Q\left(r_{N}, b, x_{0}, N_{X}\right)=r_{N} \cdot \exp \left(b \cdot\left(\frac{1}{N_{x}}-\frac{1}{x_{0}}\right)\right) \cdot\left(\frac{1}{N_{x}}\right)
$$

Where, $r_{N}$ is the weight normalization coefficient. $b, x_{0}$ are amplitude adjustment parameters. $N_{x}$ is the number corresponding to the Euclidean distance in ascending order. 
According to the principle of determining weight, the closer the distance is, the greater the weight is. But the deviation between the values can not be too big. So we adopt the standard deviation (SD) and the mean absolute deviation (MAD) to determine the weight.

$$
\begin{aligned}
& S D=\sqrt{\frac{1}{N_{q}} \sum_{i=1}^{N_{q}}\left(N_{X_{i}}-N_{X}\right)^{2}} \\
& M A D=\frac{1}{N_{q}} \sum_{i=1}^{N_{q}}\left|N_{X_{i}}-N_{X}\right|
\end{aligned}
$$

Where, $N_{x_{i}}$ represents the corresponding weight. $N_{q}$ represents the number of weight, and this paper is taken as 7. Through building the relationship between $\mathrm{SD}, \mathrm{MAD}$ and $b, x_{0}$, we can get the parameter corresponding to the minimum of the two indicators. In this paper, we determine that $b=1, x_{0}=1$.

\section{Analysis of results}

The experiment of this paper is completed based on the PC and MATLAB R2010a platform. This paper adopts the CASIA gait database, which includes three data sets: Dataset A (small library), Dataset B(multi-angle library) and Dataset C(IR library). In this paper, we use the large-scale and multi-angle Dataset B, which includes 124 individuals ( 93 male, 31 female). Each individual has 11 perspective video sequences, and each perspective includes three states of ordinary condition, coat wearing and bag wearing. Ordinary condition includes 6 video sequences, while others include 2. The size of image is $320 * 240$, and the frame rate is 25 frames/s. In this paper, the image size is normalized to $128 * 100$.

The $90^{\circ}$ gait silhouette feature is most obvious and easier to extract, so this paper analyzes the recognition performance of $90^{\circ}$ gait silhouette image under three states. In addition, this paper takes the first three sequences of ordinary condition and the first sequence of coat wearing and bag wearing as train samples, and the rest of them as test samples.

This paper adopts improved KNN to recognize the GEI, GFDEI and GFDEnI extracted under the three states, and the recognition rate is shown in table 1 :

Table 1: Recognition rate of GEI, GFDEI, GFDEnI with improved KNN (\%)

\begin{tabular}{|c|c|c|c|}
\hline Walking state & GEI & GFDEI & GFDEnI \\
\hline ordinary condition & 81.45 & 88.17 & 92.36 \\
\hline coat wearing & 80.67 & 85.48 & 85.59 \\
\hline bag wearing & 75.87 & 68.55 & 82.47 \\
\hline
\end{tabular}

It can be seen from table 1 that based on the improved KNN, the recognition rate of GFDEnI proposed in this paper is obviously better than GEI and GFDEI under the three walking states, which indicates that GFDEnI has good robustness to external condition of human walking. Furthermore, it can be seen that no matter which feature is used, the recognition rate of ordinary condition is highest, followed by coat wearing, last bag wearing, which shows the impact of external condition on human walking. The reason of higher recognition rate of GFDEnI is it introduces entropy to describe the uncertainty of gait feature in a gait period, and makes the description more accurate.

In addition, this paper takes $90^{\circ}$ GFDEnI of three states as train samples, and $54^{\circ}, 72^{\circ}, 108^{\circ}$, $126^{\circ} \mathrm{GFDEnI}$ as test samples, and the recognition rate is shown in table 2:

Table 2: Recognition rate of GFDEnI in cross- perspective

\begin{tabular}{|c|c|c|c|c|}
\hline Walking state & $54^{\circ}$ & $72^{\circ}$ & $108^{\circ}$ & $126^{\circ}$ \\
\hline ordinary condition & 60.92 & 64.11 & 68.59 & 65.13 \\
\hline coat wearing & 73.87 & 71.37 & 75.43 & 70.01 \\
\hline bag wearing & 70.66 & 80.31 & 82.62 & 72.28 \\
\hline
\end{tabular}


It can be seen from table 2 that the recognition rate is decreased with the gradual change of perspective, which indicates that the perspective has a great influence on recognition accuracy. So for the recognition of non-side perspective, the perspective problem is an important issue.

\section{Conclusions}

This paper proposes GFDEnI based on the GFDEI sequence of a complete gait period according to the Shannon entropy principle, and adopts improved KNN to recognize. GFDEnI can describe the difference between different objects accurately by taking consideration of the relationship between adjacent frames and the uncertainty of sample. Furthermore, improved KNN takes a full consideration of the impact of different samples on similarity, and sets different weight to each sample to effectively improve the recognition accuracy. Experimental results show that the algorithm this paper proposes has achieved good recognition effect. While the recognition result of cross-perspective is still not ideal, and the actual scene is always more complex, which results in some affects caused by perspective, shelter, etc. Hence, the next issue of this paper studies will be perspective problem.

\section{Acknowledgements}

This research was supported by the National Natural Science Foundation of China (Granted No. U1261114), the Xi'an University of Science and Technology Cultivation Foundation Project (2014032), the Xi'an University of Science and Technology Cultivation Foundation Project (2014033), and the Scientific Research Program Funded by Shaanxi Provincial Education Department (Program No.16JK1497) .We declare that there is no conflict of interests regarding the publication of this article and would like to thank the anonymous reviewers for their valuable comments and suggestions.

\section{References}

[1] Tracey K.M.Lee, Mohammed Belkhatir, Saeid Sanei. A comprehensive review of past and present vision-based techniques for gait recognition. Multimed Tools Application,72, pp. 2833$2869,2014$.

[2] Jia-geng FENG, Jun XIAO. View-invariant human action recognition via robust locally adaptive multi-view learning. Frontiers of Information Technology \& Electronic Engineering, 16(11),pp.917-929,2015.

[3] Liu Yudong, Su Kaina, Ma Li. A Gait Recognition Method Based on Body Skeletal Model. Computer Engineering and Applications, pp. 88-92,2005.

[4] Chen Wenjing. The study of the gait recognition based on human body joint proportion model.. Chongqing Medical University,2012

[5] Yi Meijia. Research on key techniques for gait recognition. Nanjing University of Posts and Telecommunications,2015.

[6] LIANG Shao-cong, ZHOU Ming, LI An-an. GEI based gait recognition by using KPCA and SVM. Application Research Of Computer, 7(27), pp.2798-2800, 2010.

[7] Li Rui. Gait Recognition Algorithm Research Based on Frame Difference Energy Image. Chongqing Normal University,2015.

[8] Anup Nandy, Akanksha Pathak, Pavan Chakraborty. A study on gait entropy image analysis for clothing invariant human identification. Multimed Tools Application,2016.

[9] Li Zhanli, Cui Leilei, Liu Jinxuan. Gait recognition based on collaborative representation. Application Research Of Computer, 10(33), 2015.

[10] ZHANG Rui-min, GUO Hai-gang, LI Pei-luan, Knn Algorithm Based on Kernel Method. Journal of North China Institute of Water Conservancy and Hydroelectric Power,6(28),51$52,2007$. 